\title{
Cheetah: Optimizing and Accelerating Homomorphic Encryption for Private Inference
}

\author{
Brandon Reagen*1,2, Wooseok Choi*3 ${ }^{3}$, Yeongil $\mathrm{Ko}^{4}$, Vincent T. Lee ${ }^{5}$ \\ Hsien-Hsin Sean Lee ${ }^{2}$, Gu-Yeon Wei ${ }^{4}$, David Brooks ${ }^{2,4}$ \\ *Equal contribution \\ New York University ${ }^{1}$, Facebook AI Research ${ }^{2}$, Seoul National University ${ }^{3}$ \\ Harvard University ${ }^{4}$, Facebook Reality Labs ${ }^{5}$
}

\begin{abstract}
As the application of deep learning continues to grow, so does the amount of data used to make predictions. While traditionally, big-data deep learning was constrained by computing performance and off-chip memory bandwidth, a new constraint has emerged: privacy. One solution is homomorphic encryption (HE). Applying HE to the client-cloud model allows cloud services to perform inference directly on the client's encrypted data. While HE can meet privacy constraints, it introduces enormous computational challenges and remains impractically slow in current systems.

This paper introduces Cheetah, a set of algorithmic and hardware optimizations for server-side HE DNN inference to approach plaintext speeds. Cheetah proposes HE-parameter tuning optimization and operator scheduling optimizations, which together deliver $79 \times$ speedup over state-of-the-art. However, this still falls short of plaintext inference speeds by almost four orders of magnitude. Cheetah further proposes an accelerator architecture, when combined with the algorithmic optimizations, to bridge the remaining performance gap. We evaluate several DNNs and show that privacy-preserving $\mathrm{HE}$ inference for ResNet50 can be done at near plaintext performance with an accelerator dissipating $30 \mathrm{~W}$ and $545 \mathrm{~mm}^{2}$ in $5 \mathrm{~nm}$.
\end{abstract}

\section{INTRODUCTION}

Deep learning lies at the heart of many modern services and applications, and is one of the most widely used methods to process personalized data. These models have become so successful and computationally efficient that deep learning is now integral to everyday life. However, as such services become ever-intricately woven into our lives, there is growing demand for privacy-preserving machine learning - a daunting task that this paper seeks to address.

Several techniques exist that offer privacy for deep learning inference that trade off the degree of security delivered versus computational efficiency. Generally, these techniques deliver security via system implementation or mathematical guarantees. Implementation-based methods include (i) moving computation to edge devices, i.e., local computation [54], [68], and (ii) trusted execution environments (TEEs), e.g., SGX [10], [15], [59]. Both methods achieve security by monitoring and restricting data usage via a combination of software and hardware implementations. In contrast, methods offering provable mathematical guarantees provide a theoretically-quantifiable level of privacy. Such solutions include (i) differential privacy
TABLE I: Generalization of privacy-preserving techniques.

\begin{tabular}{ccc}
\hline Solution & Security & Limitation \\
\hline Local & System & Edge performance; leaks model \\
TEE & System & Performance; side-channels \\
DP & Statistical & Applications; utility-privacy tradeoff \\
MPC & Cryptographic & Communication bandwidth \\
HE & Cryptographic & Compute \\
\hline
\end{tabular}

(DP) [4], [13], [18], [21], (ii) secure multi-party compute (MPC) [32], [36], [48], [49], and (iii) homomorphic encryption (HE) [7], [28], [31], [53]. Table I summarizes the techniques and limitations associated with each with respect to wide-scale deployment.

Each of the above solutions have differing limitations. Local execution offers individual users improved security, but there is risk of sensitive information leaking or being stolen through the model, plus model-privacy concerns for service providers [60]. TEEs have been shown to be vulnerable to side-channel attacks [15]. DP offers statistical privacy levels quantified via privacy loss $\varepsilon$ but imposes an abstruse trade-off between $\varepsilon$ and data utility [20]. Moreover, while DP has seen success in training [12], [45], its application to inference is an open question. MPC also delivers cryptographically-strong privacy guarantees. However, MPC performance is limited by communication bottlenecks [36], [40], [49], which require consideration of network-protocol and technology levels, or reformulating the algorithm itself to alleviate.

This paper focuses on homomorphic encryption (HE) to enable privacy-preserving deep learning inference, or HE inference. The key strength of HE is that it offers cryptographicallystrong privacy guarantees, but these guarantees come at the cost of massive computational overheads. These overheads are so high that existing state-of-the-art implementations of HE inference [28], [31], [53] are still five to six orders of magnitude slower than plaintext inference, i.e., unencrypted inference speed running on a CPU. To put this in perspective, the current state-of-the-art HE inference solution (Gazelle [32]) takes $800 \mathrm{~ms}$ for a single MNIST inference. These computational overheads are so extreme that prior research has yet to consider modern datasets and models, e.g., ImageNet and ResNet50, as even MNIST is currently beyond 


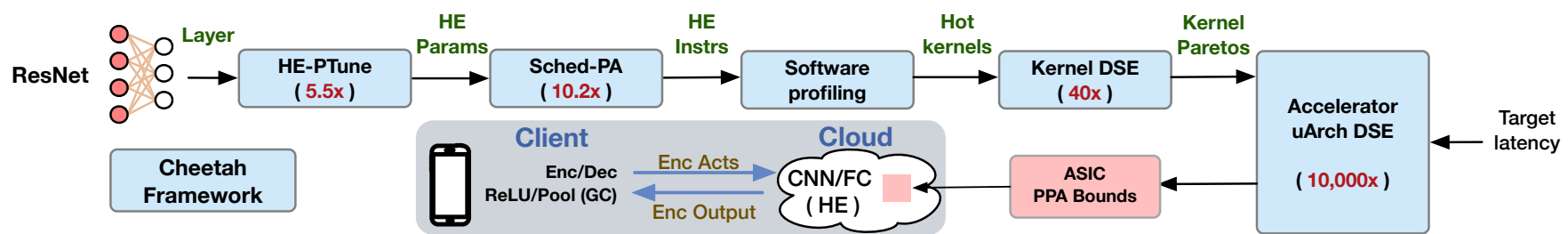

Fig. 1: The Cheetah framework and system design. Speedup achieved for ResNet50 is reported in red.

the realm of feasibility. In this paper, we propose a threepart algorithm-hardware co-design to demonstrate that the six order-of-magnitude performance gap in the state-of-the-art can be overcome.

High-performance HE inference requires addressing three key challenges. First, at the algorithmic level, HE has configurable parameters that trade performance (i.e., HE operator latency) and "computational budget," canonically know as the noise budget in HE literature. This HE noise budget limits the amount of computation (i.e., number of HE operations) that can be applied to encrypted data while still allowing correct decryption. Aggressive HE parameter setting improves performance by reducing the cost of each operation (e.g., using smaller data types), but if set too aggressively, the noise budget can be exceeded and cause the computation (i.e., decryption) to fail. The second challenge is how computations are scheduled and mapping to HE primitives. HE only supports a limited set of operators (e.g., add and multiply) that applications must be expressed as, and each operator increases noise differently. Therefore, noise-aware operator schedules can significantly improve performance by reducing accumulated noise, enabling more aggressive HE parameters to be used. The final challenge is the sheer number of computations HE inference entails. As we show, this challenge requires hardware acceleration and leveraging the extreme degrees of parallelism in both DNNs and $\mathrm{HE}$ operators to maximize performance.

Key Contributions: To address these challenges, this paper presents Cheetah, a framework (Figure 1) to enable practical HE-based privacy-preserving machine learning inference by combining algorithm optimization and custom hardware acceleration. We assume Gazelle [32], the state-of-the-art, as our baseline. Our contributions are as follows:

First, we propose HE-PTune (Section IV), which is an analytical model that tunes $\mathrm{HE}$ parameters at the algorithm level. HE-PTune automatically identifies the highestperformance HE parameter settings that satisfy noise-budget constraints by tuning HE parameters based on the needs of each layer in a deep neural network model. HE-PTune's parameter tuning yields up to $11.7 \times$ for VGG16 and $5.5 \times$ for ResNet50 performance benefit over state-of-the-art.

Second, we propose a new schedule for dot product operations called Sched-PA to minimize consumption of the noise budget and improve performance in HE. SchedPA is a partial-aligned dot product schedule, which exploits the key insight that the order of HE operations significantly impacts performance and noise budget. This allows Sched-PA to achieve a maximum additional speedup of $10.2 \times(5.2 \times$ harmonic mean) and a combined speed using HE-PTune of
$79.6 \times(13.5 \times$ harmonic mean $)$ over state-of-the-art.

Third, we propose a custom hardware accelerator architecture that combines these algorithmic optimizations to accelerate server-side HE inference to approach plaintext speeds given the abundance of parallelism and opportunities for specialization. To do this, we first conduct hot kernel profiling of an HE CPU software implementation [55] to derive the speedups necessary for plaintext latency using parameters identified by HE-PTune and Sched-PA. We also identify the amount of application inter-kernel and intra-kernel parallelism available in hot kernels. We then use these profiling results to implement a custom accelerator architecture for HE inference and conduct design space exploration for each HE kernel to measure speedups afforded by exploiting exposed parallelism.

Combining algorithmic optimizations with custom hardware acceleration, Cheetah approaches speeds comparable to plaintext inference. Cheetah is five to six orders of magnitude faster than Gazelle. For ResNet50, we find accelerator hardware requirements on the order of $545 \mathrm{~mm}^{2}$ and $30 \mathrm{~W}$ in a $5 \mathrm{~nm}$ technology node (Section VII). More importantly, we find that the accelerator area and power resources required to support HE inference at these speeds is within practical (albeit still high) resource requirements which is still on the order of a large datacenter-class GPU or similar coprocessor.

\section{OVERVIEW AND ASSUMPTIONS}

\section{A. System Setup}

A typical deep learning system setup is shown in the gray box of Figure 1. A client generates data and sends it to the cloud. The cloud performs inference and the result is returned to the client. The most direct way to apply HE is for the client to encrypt the data, the cloud processes the entire inference using $\mathrm{HE}$, and the encrypted result is returned to the client. Unfortunately, this approach has two drawbacks: (1) HE cannot readily process nonlinear functions (without incurring prohibitively large penalties) and (2) many computations in DNNs requires a relatively large HE noise budget, which necessitates larger encryption parameters, resulting in poor performance. This effect is exacerbated by deeper networks.

A classic solution is to combine multiple cryptographic solutions, as done before in [36], [38], [40], [44], [52], and partition inferences across them. The typical approach is to execute linear operators ( $\mathrm{FC} / \mathrm{CNNs})$ on the cloud using either homomorphic encryption (Gazelle [32]) or secret-sharing (MiniONN [36]), and non-linear functions on the client with Yao's garbled circuit (GC). This partition works quite well as GCs incur small computational overhead [5], [66] while the cloud can leverage powerful servers to handle the large 


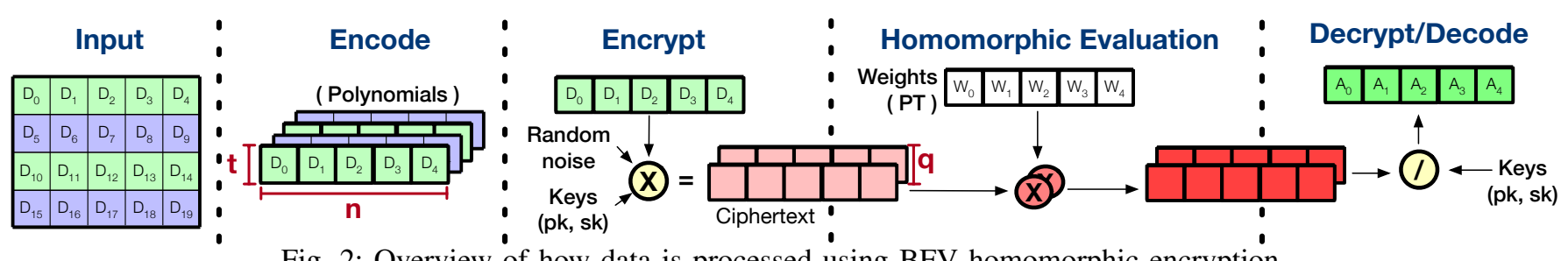

Fig. 2: Overview of how data is processed using BFV homomorphic encryption.

processing load of HE/secret sharing. In this paper, we take Gazelle as our baseline as it is the fastest known implementation of private inference and uses HE, which is the focus of Cheetah.

In Gazelle, the client encrypts data to be processed and sends it to the cloud. The cloud applies a single linear layer (e.g., convolution) to the input using HE. ReLU and pooling functions are computed on the client using a GC. The GC is configured by the cloud and sent to the client along with the encrypted linear layer outputs. The client then decrypts the outputs and processes them using the GC. Note that allowing the client to observe the original outputs after decryption can leak the cloud's private model weights (knowing the inputs and outputs of a linear function would make it trivial to steal the cloud's model). To prevent this, the cloud obscures the actual activation values (both input and output) by adding random numbers to each, i.e., the client receives encrypted activation input also obfuscated with random numbers. After decryption, the client runs the GC, which includes a subtraction circuit to remove the added random numbers securely (recovering original values), an non-linear functions (ReLU or pooling), and finally an addition to obscure the plaintext output value and protect model weights. Once GC evaluation completes, the masked output is re-encrypted by the client and sent to the cloud. On the cloud, the random numbers added to the activation are removed via $\mathrm{HE}$ subtraction and the following linear layer is computed (using HE). The HE-MPC cycle repeats for each layer of the deep network.

Note that in homomorphic encryption, decryption resets the HE noise budget. Therefore, systems like Gazelle address both issues associated with nonlinear computation and limitations of HE noise budget. However, the computational overheads of $\mathrm{HE}$ - the focus of this paper-remain prohibitive. Cheetah addresses the HE compute bottleneck, which is an architecture/hardware problem, but the proposed optimizations for HE are more generally applicable to other solutions beyond Gazelle. Solving the communication/network bottleneck is beyond the scope of this paper. We expect contributions on the algorithmic (e.g., different MPC protocols [23], [41], [44]) and technology (e.g., 5G) front to help. Therefore, Cheetah assumes the same communication overheads as Gazelle. Whenever discussing HE performance results, it is always with respect to the server-side HE inference computation.

\section{B. Threat Model}

The threat model assumed by Cheetah is the same as in Gazelle [32], and similar to other two-party compute (2PC) solutions including DeepSecure [49], MiniONN [36],
TABLE II: BFV parameters.

\begin{tabular}{cc}
\hline Parameter & Description \\
\hline$n$ & Polynomial degree (vector length) \\
$t$ & Plaintext (pt) modulus \\
$q$ & Ciphertext (ct) modulus \\
$W_{d c m p}$ & Weight (pt) decomposition base \\
$A_{d c m p}$ & Activation (ct) decomposition base \\
$\sigma^{2}$ & Variance of noise added for encryption (fixed) \\
\hline
\end{tabular}

and SecureML [40]. The model assumes the client/user and cloud are honest but curious, i.e., each agent follows the protocol precisely but may try to infer information. Under this assumption, Cheetah preserving the privacy of both the clients' data and cloud's model weights. For more details, see [32].

Note that the protocol does leak some information about the model. Because ReLU and pooling layers are performed by the client, the client can learn the number and shape of each layer. The model weights values, however, are not leaked. It is possible to obscure this information (e.g., pad tensor dimensions and add null layers), but they are not considered here and left as future work. Cheetah focuses on improving users privacy while protecting the cloud's models (considered IP today [67]) from model-stealing attacks [60].

\section{BACKGROUND}

This section provides a brief introduction to $\mathrm{HE}$ and the BFV [22]. For a complete description see [8], [22].

\section{A. Homomorphic Encryption: The Basics}

HE is a privacy-preserving encryption technique that enables computation over encrypted data, which was first shown to be possible by Gentry [24]. Since its discovery, many algorithmic improvements have been made to improve performance [6], [8], [9], [22], [25]-[27]. Modern HE schemes such as BFV allow adds and multiplies between encrypted data and derive security from the hardness of the Ring Learning With Error (RLWE) problem [37]. In BFV, noise is added during plaintext encryption and accumulates over successive ciphertext computations. If the aggregate noise exceeds a noise budget threshold, decryption fails. This noise budget is a function of the HE parameters and defines how many computations can occur before decryption fails. HE schemes of this type are called Leveled HE (LHE). In contrast, fully homomorphic encryption (FHE) schemes enable an arbitrary number of computations. FHE schemes can be built from LHE schemes via bootstrapping [22], [24]. Bootstrapping reduces the noise in the ciphertext but is expensive to implement, so most applications focus on LHE. 


\section{B. BFV: Relatively Efficient $H E$}

BFV [22] is a relatively efficient LHE scheme; Figure 2 shows an overview of the process. In BFV, data is encoded as a plaintext polynomial that is then encrypted as a pair of ciphertext polynomials. Ciphertexts are then input to addition and multiplication during evaluation. The resulting ciphertexts from evaluation are decrypted to plaintext and finally decoded to individual scalars. Polynomials are implemented as integer vectors, where the vector length (polynomial degree) and bit-width (coefficient size) are set by HE parameters. BFV parameters (listed in Table II) must be carefully tuned as they affect computational efficiency and security.

Core BFV Parameters $(n, t, q)$ : Plaintext polynomials are elements of the ring: $R_{t}=\mathbb{Z}_{t}[x] /\left(x^{n}+1\right)$, where the degree of the polynomial is less than $n$ (a power of 2). Polynomial coefficients are integers in $\mathbb{Z}_{t}$ (integers in the range $\left(-\frac{t}{2}, \frac{t}{2}\right]$ ). $t$ is called the plaintext modulus as all HE operations are taken modulo $t$ in the plaintext space. Setting $t$ requires profiling the application to ensure enough bits are used for correctness and no more, as over provisioning causes unnecessary slowdown.

Similarly, two polynomials of a ciphertext are in $R_{q}=$ $\mathbb{Z}_{q}[x] /\left(x^{n}+1\right)$, where $q$ is the ciphertext modulus. The ratio between $q$ and $t$ determines the noise budget, which sets the number of HE operators that can be computed per ciphertext before decryption fails. The ratio between $n$ and $q$ for a given variance $\left(\sigma^{2}\right)$ of Gaussian noise added for encryption sets the security strength of the HE scheme (see [22] for details).

Encoding (Packing) Data to Polynomial: When proper HE parameters are used (i.e., $t$ is prime and $t \equiv 1 \bmod 2 n$ ), a property of the ring $R_{t}$ enables a form of algorithmic parallelism. Here, each plaintext polynomial in $R_{t}$ and, hence, the ciphertext, can be packed with $n$ data. This means that each $\mathrm{HE}$ addition or multiplication can actually perform an $n$-way parallel element-wise computation. With packing, each scalar data is tied to a slot, and slots can be thought of as individual elements in the integer array. Packing significantly improves HE performance; $n$ is typically on the order of thousands and the benefits of packing are proportional [58].

Polynomial Representations: Polynomials are represented in two spaces-coefficient and evaluation. The coefficient representation is how polynomials are typically represented, e.g., $\sum_{i=0}^{n-1} \alpha_{i} x^{i}$. The evaluation space is analogous to the frequency domain of time-domain signals. Similar to FFT, efficient conversion between the two is done via the Number Theoretic Transform (NTT) [9], [58]. Cheetah keeps polynomials in the evaluation space and converts to coefficient space only as needed for operations like decomposition (see below). Using the evaluation space as a default representation reduces the number of NTTs needed for homomorphic CNN/FC. Note that applying NTT to ciphertexts does not affect noise.

1) Operations of BFV: BFV consists of three operators: $\mathrm{HE} \_\mathrm{Add}, \mathrm{HE} \_\mathrm{Mult}$, and HE_Rotate. Recall that the HE_Add and HE_Mult operate on vectors of packed data, so they are effectively SIMD-add and SIMD-multiply operations. Note that the underlying implementations of $\mathrm{HE}$ _Add and HE_Mult consist of many modular arithmetic
TABLE III: Impact on Noise of basic BFV operations.

\begin{tabular}{cc}
\hline & Noise Bound after Each Operation \\
\hline Noise $\left(v_{0}\right)$ in fresh ct & $2 n B^{2}(B=6 \sigma)$ \\
$\mathrm{HE}$ Add $\left(\mathrm{ct}_{0}, \mathrm{ct}_{1}\right)$ & $v_{0}+v_{1}$ (additive) \\
$\mathrm{HE}$-Mult $\left(\mathrm{pt}, \mathrm{ct}_{0}\right)$ & $n l_{p t} W_{d c m p} v_{0} / 2$ (multiplicative) \\
$\mathrm{HE}$-Rotate $\left(\mathrm{ct}_{0}\right)$ & $v_{0}+l_{c t} A_{d c m p} B n / 2$ (additive) \\
\hline
\end{tabular}

TABLE IV: HE-PTune performance models.

\begin{tabular}{ccc}
\hline $\mathrm{CNN}$ & $\mathrm{HE} \_\mathrm{Mult}$ & $\mathrm{HE} \_$Rotate \\
\hline$n \geq w^{2}$ & $l_{p t} c_{i} c_{o} f_{w}^{2} / c_{n}$ & $c_{i} c_{o} f_{w}^{2} / c_{n}$ \\
$n<w^{2}$ & $l_{p t}\left(2 c_{n}-1\right) c_{i} c_{o} f_{w}^{2}$ & $\left(2 c_{n}-1\right) c_{i} c_{o}\left(f_{w}^{2}-1\right)$ \\
\hline $\mathrm{FC}$ & $\mathrm{HE} \_\mathrm{Mult}$ & $\mathrm{HE} \_$Rotate \\
\hline$n \geq n_{i}, n \geq n_{o}$ & $l_{p t} n_{i} n_{o} / n$ & $n_{i} n_{o} / n-1+\log \left(n / n_{o}\right)$ \\
$n \geq n_{i}, n<n_{o}$ & $l_{p t} n_{i} n_{o} / n$ & $\left(n_{i}-1\right) n_{o} / n$ \\
$n<n_{i}, n \geq n_{o}$ & $l_{p t} n_{i} n_{o} / n$ & $\left(n_{o}+\log \left(n / n_{o}\right)\right) n_{i} / n$ \\
$n<n_{i}, n<n_{o}$ & $l_{p t} n_{i} n_{o} / n$ & $(n-1) n_{i} n_{o} / n^{2}$ \\
\hline
\end{tabular}

calculations, different from a single cycle integer add or multiply computation. Table III shows the amount of noise introduced by each operator which depend on BFV parameters. $B$ is the bound of the noise added during encryption while $v_{i}$ represents the noise in ciphertext $\mathrm{ct}_{i}$. The remaining parameters $\left(l_{p t}, l_{c t}, W_{d c m p}\right.$, and $\left.A_{d c m p}\right)$ are for decomposition, defined in Section III-B2.

HE_Add: Two ciphertexts can be added homomorphically by summing each ciphertext coefficient followed by a modulo operation. I.e., a resulting coefficient outside the range $\mathbb{Z}_{q}$ is reduced to be in $\mathbb{Z}_{q}$. Reduction is implemented as a comparison and subtraction to keep the performance overhead low. Each $\mathrm{HE}$ _Add operation increases noise additively.

HE Mult: BFV supports both ct-ct and pt-ct multiplication. Cheetah uses pt-ct multiplication to multiply plaintext weights by encrypted activations. Pt-ct multiplication is achieved by multiplying evaluation space ciphertext polynomials by the evaluation space plaintext polynomial containing weights on a per-element basis. Performance is limited by the modular reduction required for each polynomial coefficient of output. Cheetah uses Barret reduction (see Section IV-A). HE_Mult operations increases noise multiplicatively.

HE_Rotate: BFV supports slot rotation within a packed polynomial to enable computation between data in different slots. Since HE_Add and HE_Mult are element-wise operations, computations like dot products require $\mathrm{HE}$ Rotate to align partial products and implement the reduction (see Section V-A). HE Rotate is computationally expensive with many steps, and increases noise additively. We refer the reader to [9], [62] for details.

2) Polynomial Decomposition: Decomposition is used to segment polynomials into multiple components with smallervalued coefficients. The key idea is that HE operations over smaller coefficient polynomials reduces noise growth. To enable this, Cheetah has two parameters for polynomial decomposition: $W_{d c m p}$ and $A_{d c m p}$ (Table II), which defines the base that polynomials are decomposed to. Decreasing decomposition base increases the number of decomposed polynomials which decreases operator noise growth but increases the total 
TABLE V: Noise models for CNN and FC layer.

\begin{tabular}{cc}
\hline CNN & Output Noise \\
\hline$n \geq w^{2}$ & $f_{w}^{2} c_{i} \eta_{M} v_{0}+\eta_{A} c_{i}\left(f_{w}^{2}-1+\left(c_{n}-1\right) / c_{n}\right)$ \\
$n<w^{2}$ & $\left(2 f_{w}-1\right) f_{w} c_{i} \eta_{M} v_{0}+\eta_{A} c_{i}\left(2 f_{w}+1\right)\left(f_{w}-1\right)$ \\
\hline FC & Output Noise \\
\hline$n \geq n_{i}$ & $n_{i} \eta_{M} v_{0}+\eta_{A}\left(n_{i}-1\right)$ \\
$n<n_{i}$ & $n_{i} \eta_{M} v_{0}+\eta_{A} n_{i}(n-1) / n$ \\
\hline
\end{tabular}

amount of compute. Once decomposed operators complete, resulting segments are summed to get the final result.

$\mathrm{HE}$ _Rotate requires ciphertext decomposition, otherwise a single operation can exceed the noise budget. The decomposition base $A_{d c m p}$ is used to factor ciphertext polynomials into multiple, smaller-magnitude polynomials when $\mathrm{HE}$ _Rotate is applied. We denote $l_{c t} \approx \log _{A_{d c m p}}(q)$ as the number of polynomials with base $A_{d c m p}$ resulting from the decomposition. Since $\mathrm{HE}$ _Rotate noise increase is additive, with decomposition noise increase by an additive factor proportional to $A_{d c m p}$ and the increase in number of polynomial operations $l_{c t}$.

$\mathrm{HE}$ _Mult also benefits from decomposition to reduce noise. For neural networks, we use HE_Mult with decomposition to compute the partial products since weights are presented in plaintext. Using a decomposition base $W_{d c m p}$, the plaintext polynomial can be decomposed into $l_{p t} \approx \log _{W_{d c m p}}(t)$ polynomials. The resulting $\mathrm{HE} \_\mathrm{Mult}$ with decomposition requires $l_{p t}$ polynomial multiplications to implement but reduces noise growth by a factor of around $t /\left(l_{p t} W_{d c m p}\right)$.

\section{HE-PTune: Models \& PARAmeter Tuning}

$\mathrm{HE}$ parameter selection is a major source of complexity (i.e., $\left.n, t, q, W_{d c m p}, A_{d c m p}\right)$, where proper selection strikes a balance between noise budget and performance. A greater noise budget enables more computations per ciphertext but slower HE operators. Existing solutions rely on over-provisioning noise budgets, resulting in suboptimal performance. This section proposes HE-PTune: analytical performance and noise models for $\mathrm{HE} \mathrm{DNN}$ operators to maximize performance via finegrained parameter tuning. Tuning parameters with HE-PTune delivers up to a $11.7 \times$ speed up over the state-of-the-art.

\section{A. Performance Modeling}

HE-PTune's performance model analytically derives the total number of underlying integer multiplications per layer. (Recall that the $\mathrm{HE}$ operator $\mathrm{HE}$ _Mult consists of many integer multiplications.) Most HE operators resolve to multiplication operations and ones that do not have run-times either strongly correlated or dominated by those that do. Performance models for $\mathrm{CNN}$ and FC layers are built by first capturing all HE and NTT operations. Then all operations are reduced to the total number of underlying integer multiplication operations.

1) Modeling CNNs: $\mathrm{CNN}$ layers are parameterized as $\left(w, f_{w}, c_{i}, c_{o}\right)$, where $w^{2}$ and $f_{w}^{2}$ represent the size of input image and weight filter, and $c_{i}$ and $c_{o}$ denote the number of input and output channels. Encryption parameters follow the notation defined in Table II. Effective modeling of
$\mathrm{HE} \_\mathrm{Mult}$ and $\mathrm{HE} \_$Rotate counts require consideration of two cases: 1) the ciphertext slot count is greater than an input image (i.e., $n \geq w^{2}$ ), 2) the ciphertext slot count is less than an input image (i.e., $n<w^{2}$ ). We use $c_{n}$ to model the relationship between ciphertext slots and input image size. $c_{n}$ is defined as the number of input image channels per ciphertext (i.e., $n / w^{2}$ ) in the first case and the number of ciphertexts per input image channel $\left(w^{2} / n\right)$ in the second. Table IV shows how each case counts the number of $\mathrm{HE}$ operations per CNN layer.

$\mathrm{HE}$ Rotate operations require both polynomial multiplication and NTTs. Precisely, assuming a ciphertext decomposition base $A_{d c m p}, 2 l_{c t}$ multiplications and $l_{c t}+1$ NTT $\left(l_{c t} \approx \log _{A_{d c m p}} q\right)$ are required per $\mathrm{HE}$ _Rotate. Each $n$-point NTT entails $n \log n / 2$ butterflies. Cheetah uses Harvey's butterfly ( 3 integer-multiplications per butterfly). HE_Mult does not require NTTs as in Cheetah the default polynomial representation is the evaluation space. Each $\mathrm{HE}$ _Mult requires two element-wise modular multiplications between the two polynomials, resulting in $2 n$ modular multiplications per $\mathrm{HE}$ _Mult. Cheetah uses Barrett reduction [33], which uses five integer-multiplications per reduction.

2) Modeling FCs: A similar process is repeated to model FC layers. The required number of integer multiplications per $\mathrm{HE}$ Mult and $\mathrm{HE}$ Rotate operations is the same in both $\mathrm{CNN}$ and $\mathrm{FC}$, the only difference is the number of $\mathrm{HE}$ Mult and $\mathrm{HE}$ Rotate counts. Here, an FC layer is parameterized as $\left(n_{i}, n_{o}\right)$, where $n_{i}$ and $n_{o}$ represent the number of input and output activations. The required number of $\mathrm{HE}$ Mult and $\mathrm{HE}$ Rotate for all possible cases are summarized in Table IV.

\section{B. Noise Modeling}

Once CNN/FC layers are implemented as $\mathrm{HE}$ operations (see Section V-A), noise growth can be modeled using the equations in Table III. We developed a model for layer noise as a function of both $\mathrm{HE}\left(n, t, q, W_{d c m p}, A_{d c m p}\right)$ and DNN $\left(f_{w}, w, c_{i}, c_{o}\right.$ for $\mathrm{CNN}$ and $n_{i}, n_{o}$ for FC) parameters. Note that directly applying the equations in Table III estimates noise in the worst case. The worst case is very rare (see below) and causes unnecessarily slow HE parameters to be selected. To overcome this, we develop practical noise estimations for $\mathrm{HE}$ operators and provide a theoretical analysis of the decryption failure rate. We also note that all prior work on high-performance HE [11], [28], [32] sets HE parameters using heuristics, providing high-likelihoods for success but not guaranteeing it.

Cheetah builds a theoretically-motivated, empiricallyderived noise model that minimizes computational overheads for a targeted probability of success. We observe that added encryption noise is sampled from an independent bounded discrete Gaussian (IBDG) distribution with variance $\sigma^{2}$, and if $X_{i}^{\prime}$ 's are IBDG with variance $\sigma_{i}^{2}$, then $\sum_{i} \alpha_{i} X_{i}$ is also IBDG with variance $\sum_{i} \alpha_{i}^{2} \sigma_{i}^{2}$. As the noise grows multiplicatively in

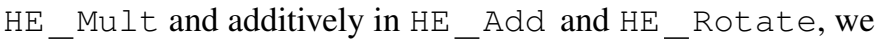
can compute the variance of the output noise after each layer under the independence assumption, which was validated in 


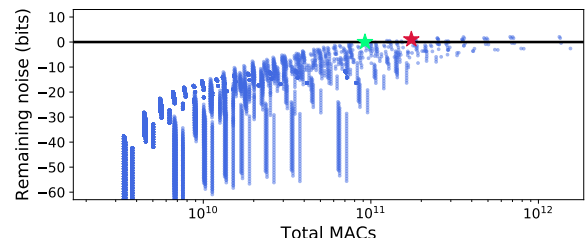

(a) Layer5 (FC)

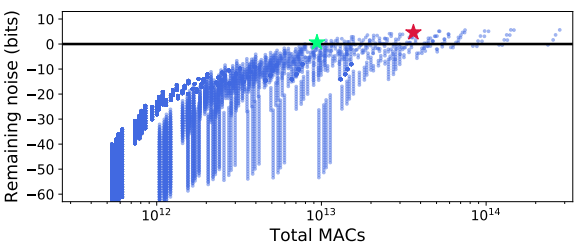

(b) Layer0 (CNN)

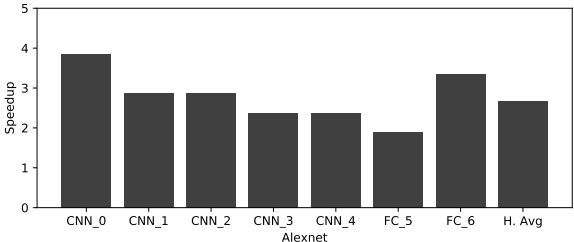

(c) Alexnet Speedup

Fig. 3: Comparison of HE-PTune and Gazelle using AlexNet. Blue dots are HE configurations modeled by HE-PTune. The red star is Gazelle's configuration and the green star is the optimal found by HE-PTune. Layer5 and Layer0 show the best and worst configuration for Gazelle with respect to utilized noise budget. HE-PTune's speedup for all layers on the right.

[19]. Then, since the output noise $(Y)$ is IBDG with standard deviation $\left(\sigma_{Y}\right)$, the probability of decryption failing is bound by $\operatorname{Pr}(|Y| \geq q /(2 t)) \leq 2 \exp \left(-q^{2} /\left(4 t^{2} \sigma_{Y}^{2}\right)\right)$. We use these equations to derive an output noise threshold for a probability of correct decryption. Therefore, instead of using worst-case bounds and guaranteeing correct decryption, our noise model uses the scaled expressions given in Table III. Cheetah uses a scaling factor $c$ such that the decryption failure rate is provably less than $10^{-10}$, which is negligible as it is much lower than the DNN's misclassification rate.

The noise models are given in Table V. Here, $v_{0}$ is the initial noise for the input ciphertext, $\eta_{M}$ is the noise due to HE_Mult, and $\eta_{A}$ is the growth factor from HE Rotate. By dividing $\frac{q}{2 t}$ by the output noise (and taking the log), the remaining noise budget in bits is given. When the budget is negative, decryption fails; when positive, it fails with probability $\leq 10^{-10}$.

\section{HE Parameter Space Exploration}

Using a single set of HE parameters for all DNN layers results in poor performance, as HE parameters are provisioned for the worse case layer noise. Using HE-PTune's models for noise and performance, parameters can be readily tuned on a per-layer basis. HE-PTune takes layer hyperparameters as input and outputs optimal HE parameters found via a design space exploration. Because the model is analytical, a vast parameter space can be explored in a matter of minutes.

An example of HE parameter space exploration are given in Figure 3 for AlexNet on ImageNet. Each blue dot is unique set of HE parameters modeled with HE-PTune to estimate computation and remaining noise budget. Red stars indicate parameters used by Gazelle and green stars show the optimal point found using HE-PTune. Gazelle uses the same sets of HE parameters for all layers. Of all layers in the model, Layer 5 has the smallest remaining noise budget, and it follows that the speedup between Gazelle and Cheetah is the lowest for this layer (see bars in Figure 3). Using HE-PTune, empirical results show using a single set of parameters is inefficient and unnecessary. The highest Cheetah speedup is in Layer 0, where Gazelle has an excess noise budget of 4.6 bits whereas HE-PTune finds a configuration leaving only 1 bit of noise budget. Improvements come from tailoring parameters to the requirements of each layer.
HE-PTune also eases the finding functional HE parameter settings in the first place. Recall that any point where the noise budget is exceeded fails to decrypt. Of all the points evaluated in the design space search, over $99 \%$ have a negative remaining noise budget and will not work. Finding HE parameters is difficult, and further motivating HE-PTune.

We validate the parameter sets from HE-PTune using different $\mathrm{CNN}$ and FC layers used in popular DNNs, including: LeNet-300-100 and LeNet5 for MNIST [35], and AlexNet [34], VGG16 [57], and ResNet50 [30] for ImageNet [51]. Each layer is tested using a variety of $\mathrm{HE}$ parameters, with no consideration of noise budget to explore the parameter space. Execution times are collected by implementing each CNN/FC layer in the SEAL HE library [55] and measuring its performance on a Xeon server. The remaining noise budget is collected after each run using SEAL's internal measuring capability and API. Overall, we find that due to the underlying randomness of the noise, the noise model shows slightly larger error than the performance model. However, this is acceptable as the worst-case errors are within 1 bit in the low-remaining noise budget region of the space.

\section{Partial-Aligned SCHEduling}

This section introduces a new dot product schedule - SchedPA - to improve HE performance on FC and CNN layers. Recall that each HE primitive has different run-time and additive noise trade-offs (Section III) and the overheads of different primitive schedules are not associative so order of operations matters. Operation orderings with less noise are beneficial as it enables higher-performance via more computationally efficient HE parameters. As a result, Cheetah's Sched-PA dot product optimization provides a performance benefit of up to $10.2 \times$ compared to prior work.

\section{A. Sched-PA: Partial-Aligned Dot Products}

The key challenge for implementing HE dot products is optimizing how data is packed into polynomial slots and the relative order of operations. Computing a dot product in $\mathrm{HE}$ requires all three primitives: $\mathrm{HE}$ _Mult, $\mathrm{HE}_{-} \mathrm{Add}$, and HE_Rotate. Partial products are computed using an HE_Mult operation between a ciphertext (encrypted activation) and a plaintext (model weights). Each partial is accumulated with a series of $\mathrm{HE}$ _Add operations to reduce the final output. The challenge is that HE operations only 


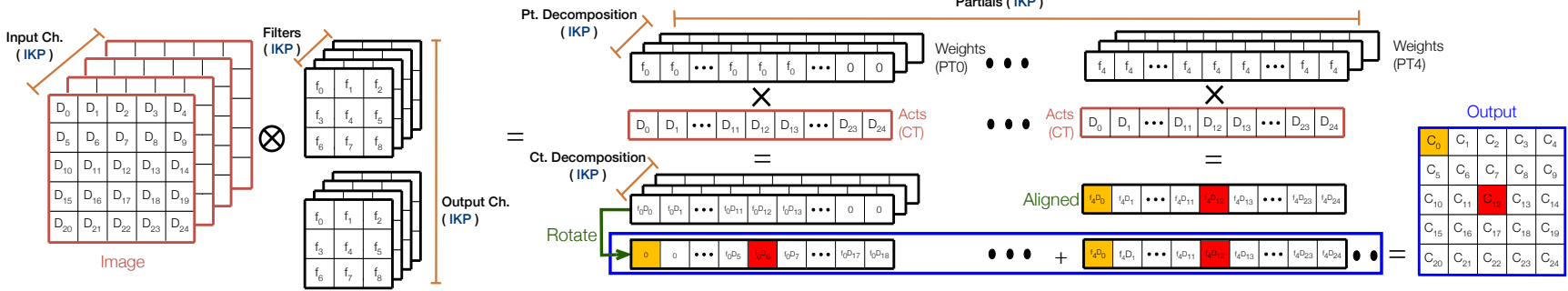

Fig. 4: How Cheetah implements CNNs using Sched-PA. Sources of inter-kernel parallelism (IKP) are labeled.

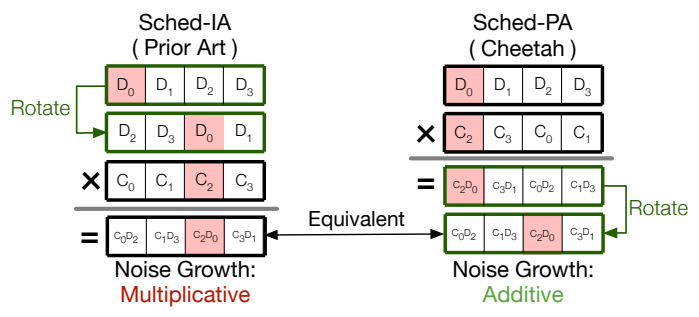

Fig. 5: Sched-IA (input-aligned) versus Sched-PA (partialaligned) dot product schedules. Cheetah uses Sched-PA to improve performance of CNN and FC layers.

support computation between aligned polynomial slots. This means that when polynomial $\mathrm{A}$ and $\mathrm{B}$ are multiplied (resulting in $\mathrm{C}), \mathrm{C}[\mathrm{i}]=\mathrm{A}[\mathrm{i}] \times \mathrm{B}[\mathrm{i}], \forall i \in[0, n)$. To properly reduce each of the partials of a dot product, the slots in $\mathrm{C}$ must be aligned to use the correct values.

Prior work aligns the inputs before performing multiplication, referred to here as an input-aligned schedule (SchedIA) [29], [32]. In Sched-IA, the input ciphertext is first aligned, or rotated, to the correct output slot, and plaintext weights are packed appropriately. The post-rotation ciphertext and plaintext are then multiplied, resulting in a dot product partial (ciphertext). Resulting partial ciphertexts can be readily accumulated to compute the final value.

Cheetah proposes a new dot product implementation called Sched-PA (see Figure 4, 5). Our key insight is that $\mathrm{HE}$ _Mult increases noise by a multiplicative factor $\eta_{M}$ $\left(\leq \bar{n} l_{p t} W_{d c m p} / 2\right)$ whereas $\mathrm{HE}$ Rotate is additive $\eta_{A}$. In Sched-PA, the initial input ciphertext is always kept in its original order. Weights are again packed into a plaintext polynomial and aligned with ciphertext slots to compute the correct partial product via $\mathrm{HE}$ Mult. Finally, resulting partial product ciphertexts are aligned such that the partial slot matches the correct output slot. Figure 5 also shows Sched-PA compared to the other approach.

The benefit stems from noise accumulation in chained HE operations. Recall that $v_{0}$ and $\eta_{A}$ represent the initial input ciphertext noise and additive noise from $\mathrm{HE}$ Rotate, respectively. Thus, a dot product using the partial aligned schedule experiences a noise growth of $\eta_{M} v_{0}+\eta_{A}$. In contrast, the Sched-IA dot product first rotates then multiplies, resulting in noise growth of $\eta_{M}\left(v_{0}+\eta_{A}\right)$, significantly larger than Sched-PA. Saving noise enables HE-PTune to identify higher performance HE parameter settings, ultimately resulting in performance benefit.

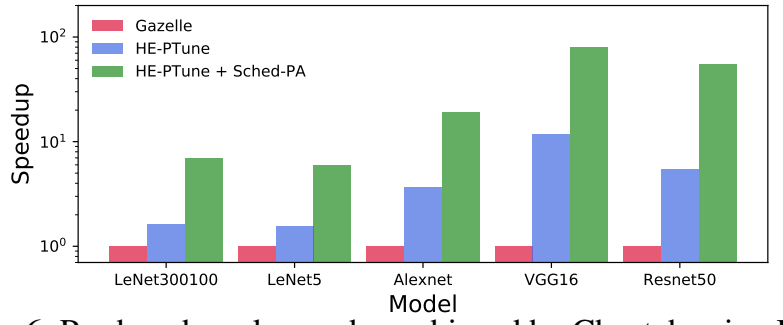

Fig. 6: Per-benchmark speedup achieved by Cheetah using HEPTune and Sched-PA. Speedup is relative to Gazelle [32].

\section{B. Implementing Low-Noise Convolution}

Figure 4 shows an example of how CNNs are implemented in HE using Sched-PA. FC layers follow precisely the same steps as CNNs, as the core primitives are also dot products. First, the input activation ciphertext (Acts) is encoded by placing adjacent pixels from the client's image sequentially in polynomial slots. This ordering eases partial ciphertext alignment. Next, CNN filter weights (Filter) are encoded into plaintext polynomials. Each activation-weight polynomial is multiplied with $\mathrm{HE}$ _ Mult to compute the partials. The resulting partial polynomials are then rotated to align partial slots to the proper output-neuron slot. Finally, with all partials computed and aligned, the ciphertexts are reduced with $\mathrm{HE}$ Add. Note how polynomial slots allow multiple output neurons to be computed in single ciphertext. This algorithmic parallelism provides substantial performance and memory savings for $\mathrm{HE}$ as without it, each thousand degree polynomial would only compute a single output neuron.

The zeros found in weight plaintext slots (e.g., PT0) ensure the correct computation. For example, the red slot in Figure 4 shows how accumulation works. After $f_{0}$ is multiplied to $D_{6}$ in the first $\mathrm{HE}$ Mult, the result is rotated right 6 times to be accumulated in the red slot $\left(C_{12}\right)$. When this rotation is performed, $D_{19}$ aligns to slot 0 , however $f_{0} D_{19}$ should not be accumulated in the output of slot 0 (i.e., $C_{0}$ ). Selectively adding zeros in the plaintext slots avoids this boundary case.

\section{Evaluation Results}

The effectiveness of Sched-PA is evaluated using five standard CNN models. HE-PTune is employed to maximize benefits and tune HE parameters on a per-layer basis. Multiple experiments are run to show the benefits of HE-PTune and Sched-PA independently and relative to Gazelle.

The results for each model are shown in Figure 6. Overall, the Cheetah optimizations substantially outperform Gazelle. 


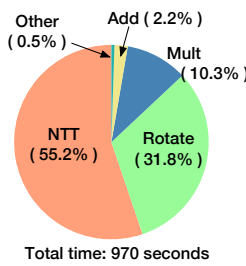

(a) Time breakdown

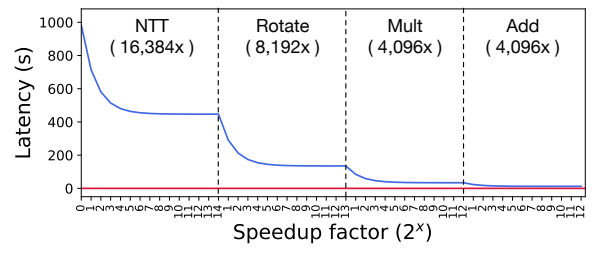

(b) Speedup Needed

Fig. 7: Profiling results for ResNet50 and speedup needed by each kernel to match plaintext inference latency.

Using the harmonic mean, $2.98 \times$ speedup comes from HEPTune alone $(5.25 \times$ ignoring MNIST). Sched-PA provides an additional speedup of $5.20 \times(6.11 \times$ ignoring MNIST $)$ for a total mean performance improvement of $13.5 \times$ and maximum of $79.5 \times$ over Gazelle $(30.3 \times$ mean without MNIST).

Significant performance overheads are incurred by Gazelle as Sched-IA requires substantial ciphertext and plaintext decomposition. Each time a polynomial is decomposed to reduce noise, the number of polynomials that must be computed grows proportionately. In ResNet50, Cheetah's optimizations result in a ciphertext decomposition base of 8 to 16 more bits. A higher ciphertext decomposition bases result in fewer decomposed polynomials for HE Rotate, and substantial performance improvements. With Sched-PA, Cheetah avoids all plaintext decomposition.

\section{Profiling HE Inference}

HE-PTune and Sched-PA significantly improve the performance over the state-of-the-art [32], e.g., 55.6× for ResNet50. However, with these optimizations alone HE inference is still 3-4 orders of magnitude slower than plaintext inference, i.e. unencrypted inference on a CPU. To better understand performance bottlenecks we profile a software implementation of HE inference and compute the speedup needed from hardware acceleration.

We implement HE ResNet50 in the SEAL library [55]. Using Cheetah to tune parameters and maximize performance, one HE inference takes 970 seconds on an Intel Xeon E5-2667 server. The same unencrypted inference (on the same server) takes 100 milliseconds using Keras [14]. Since SEAL only supports CPUs, we perform profiling on the CPU platform. Below we profile NTT running on a GPU.

Profiling results are summarized in the pie chart of Figure 7. Notice that only a few kernels dominate performance (HE_Mult, HE_Add, HE_Rotate, and NTT). HE Rotate in Figure $\overline{7}$ does not include NTT as this is shown separately. Of the four, NTT is the primary bottleneck taking $55.2 \%$ (535 seconds) of the run time. The SEAL profile also contains a long-tail of small functions, labeled "Other" in Figure 7. We note that most of the "Other" function time is in construction/destruction.

Using the profiling results, we compute the speedup needed from each HE kernel to achieve plaintext inference latency. Figure 7 shows the limit study results and how various speedup factors impact overall run time. The $\mathrm{x}$-axis shows the speedup

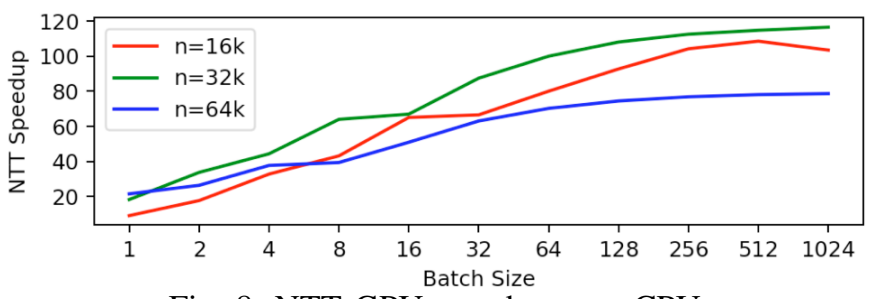

Fig. 8: NTT GPU speedup over CPU.

factor applied to each kernel function (note log scale); the final speedup factor for each kernel is the speedup needed (e.g., 16,384 for NTT). The y-axis shows absolute latency. From left to right, the plot shows how the total inference latency decreases as each theoretical speedup factor is applied to each function. Kernel speedup is applied successively where the run time from the most aggressive speedup factor is taken as the base for the next function. The horizontal red line is the $100 \mathrm{~ms}$ plaintext inference latency target.

Speeding up HE with GPUs: One way to improve kernel performance is with GPUs. To understand the the limitations of HE on GPUs, we benchmark NTT, the primary HE bottleneck, using the cuHE library [16] on an NVIDIA 1080-Ti GPU. GPU speedup is reported for different NTT batch sizes (1 to 1024 ) and vector lengths $n=16 \mathrm{~K}, 32 \mathrm{~K}$, and $64 \mathrm{~K}$ (Figure 8). At larger batch sizes (512/1024), the speedup saturates at $120 \times$. The nvprof profiler shows that for a batch size of 512, the GPU is utilized with $70 \%$ warp occupancy and $85 \%$ warp execution efficiency.

Other first order limitations to performance likely derive from (a) non-native, long integer data types requiring emulation, (b) modular arithmetic, which adds branch instructions and over 10 compute instructions per multiplication. Despite the speedups, GPUs fall well short of the improvements required to reach native $\mathrm{CPU}$ speed.

\section{HE INFERENCE ACCELERATOR ARCHITECTURE}

This section proposes a general accelerator architecture for $\mathrm{HE}$ inference to bridge the remaining performance gap.

\section{A. Accelerator Architecture}

The proposed accelerator architecture is shown in Figure 9. At a high level, it is composed of ciphertext (CT) processing engines (PEs) that receive data from a PCIe-like streaming interface and buffer intermediary results in SRAMs (Figure 9a). Hierarchically, PEs are composed of partial processing lanes or Lanes, and a partial reduction network, which implement the HE dot product (Figure 9b). Lanes are further decomposed into individual HE operators (Figure 9c).

1) PEs: Output Neuron Engines: Our architecture is designed to maximize performance and parallelism by being output-stationary. Each PE processes a single ciphertext of output neurons and all compute-memory resources necessary for the output remain local to the PE; the number of PEs is parameterized. When there are more output neurons per layer (Parallel Output CTs) than physical PEs, we time multiplex the computation across multiple PE executions. The PEs are connected to input and output buffers used to route data to 


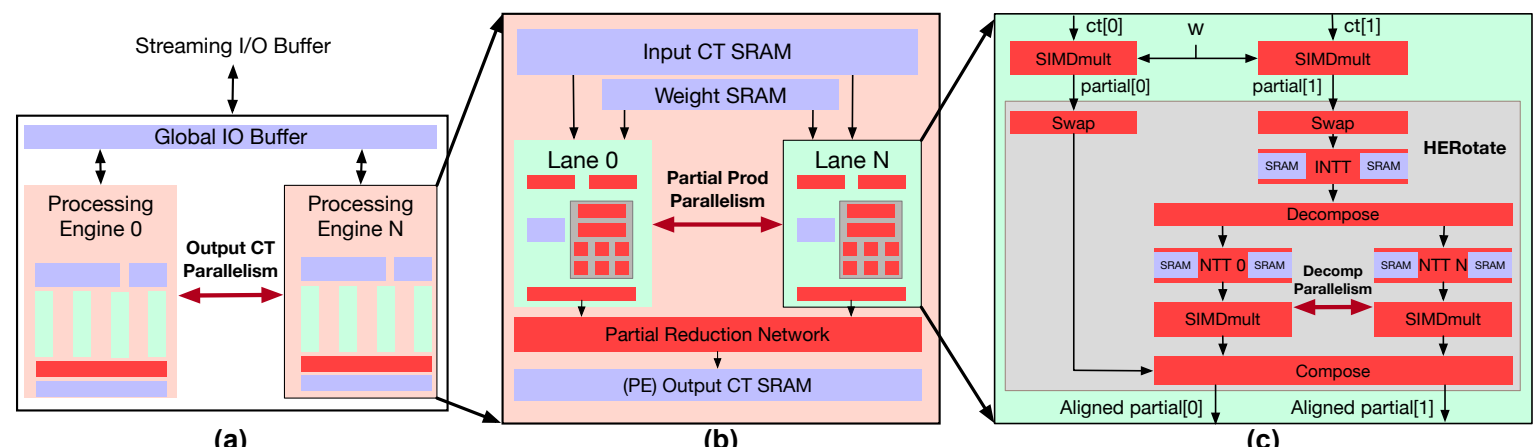

(a)

(b)

(c)

Fig. 9: Cheetah accelerator architecture. (a) The accelerator is composed of parallel PEs operating in output stationary fashion. Off-chip data is communicated via a PCIe-like streaming interface, and data is buffered on-chip using global PE SRAM. (b) Each PE contains Partial Processing Lanes which compute the HE dot product. (c) Lanes comprise individual HE operators.

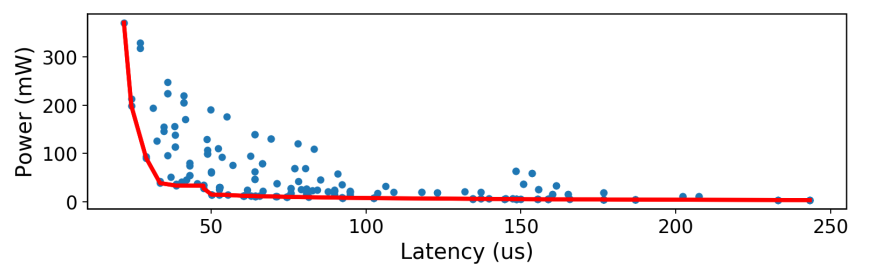

Fig. 10: DSE for NTT; Pareto frontier in red.

and from the host. These buffers constitute small SRAMs as they only handle communication, all state and intermediates are local to PEs.

The internals of the PE contain partial processing lanes and reduction networks. Each PE contains an Input CT buffer to store a copy of all activation CTs locally, a relatively small SRAM for weights, a set of partial processing lanes, a partial reduction network, and output CT SRAM. Each Lane is capable of processing a unique dot product partial; the number of lanes is parameterizable. Lanes within a PE operate in lockstep to enable aggressive reuse of twiddle factor SRAMs required for NTTs. The partial reduction network is configured based on the number of partials computed in parallel (i.e., number of Lanes). Input CT SRAMs are provisioned with enough capacity to hold all the inputs with sufficient bandwidth to feed all Lanes.

2) Lanes: Partial Engines: Lanes are the backbone of the accelerator and implement the HE operators. In Figure 9c, HE kernel blocks are denoted in red. Intermediary SRAMs, shown in blue, are used to store results between HE kernels. We use SRAMs instead of off-chip DRAM for intermediary results because of the high internal bandwidth required within NTT modules to support aggressive parallelism. In the worst case, each NTT kernel requires $13 \mathrm{~GB} / \mathrm{s}$ of combined internal bandwidth; each lane contains multiple NTTs and each PE contains many lanes. Aside from the NTT kernels that require a strided access pattern, all operations within a Lane can be made streaming (i.e, no SRAMs needed after kernels). This allows the architecture to save SRAM resources. The NTT activation decomposition factor $A_{d c m p}$ introduces a parametrizable degree of inter-NTT parallelism, which otherwise does not impact overall latency. For high-performance, enough lanes are allocated to execute all partials in parallel.
The lane architecture shows the datapath and dependencies to compute a single partial dot product. Both input polynomials (CT[0] and CT[1]) are first multiplied by plaintext weights using the $\mathrm{HE}$ Mult operator, outputting partial polynomials. The datapaths diverge as BFV splits the compute asymmetrically between partial[0] and partial[1]. HE_Rotate is applied to perform polynomial slot alignment. For partial[1], inverse NTT (INTT), decomposition, NTT, and composition units are applied. The datapath for partial[1] splits after the INTT computation in order to implement ciphertext decomposition. Recall that decomposition reduces noise growth; however, the trade-off manifests here as additional compute requirements. Fortunately, the additional computation can be parallelized (NTTs and SIMDmults). The decomposed polynomials are then converted back to the evaluation domain and combined with swapped partial[0] to produce the aligned partial that is fed to the partial reduction network in the PE, which consists of SIMDadd units.

\section{EXPERIMENTAL RESULTS}

This section presents design space exploration results of the parameterized accelerator architecture. We show that by combining Cheetah's algorithmic and architectural optimizations provides near plaintext performance.

\section{A. Methodology}

Design space exploration consists of sweeping various accelerator microarchitectural parameters. Each kernel ( $\mathrm{HE} \_\mathrm{Mult}, \mathrm{HE} \_$Add, and $\mathrm{HE}$ _ Rotate-which is split into Swap, INTT, Decompose, NTT, SIMDMult, and Compose) is built using Catapult HLS 10.3d [1] and synthesized with a commercially-available 40nm standard cell library targeting $400 \mathrm{MHz}$. For each kernel, we evaluate hundreds of design points to explore different design tradeoffs and identify optimal implementations. Each kernel accelerator's microarchitecture is parameterized by memory bandwidth (or IO in the case of streaming kernels), datapath parallelism (i.e., hardware loop unrolling), pipelining (i.e., initiation-interval), and clock frequency. We estimate power, performance, and area using Catapult's output RTL and power analysis flows. We use a commercial SRAM compiler to compile each SRAM 


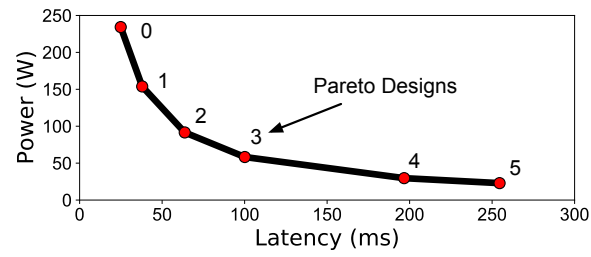

(a) ResNet50 DSE

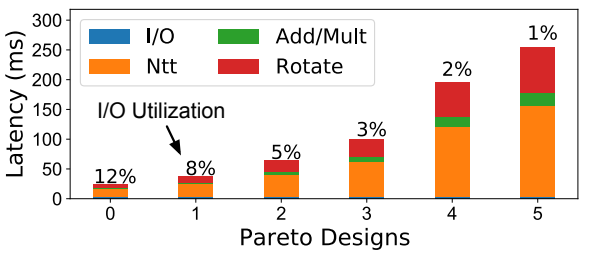

(b) Time Breakdown

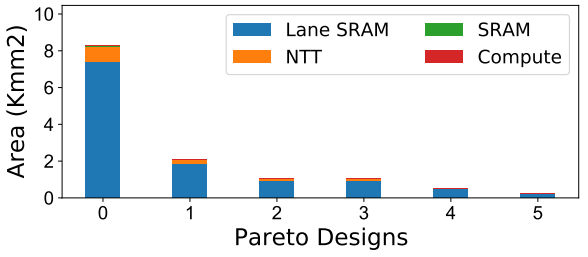

(c) Area Breakdown

Fig. 11: (a) Power-latency Pareto for ResNet50 DSE. (b) Run time breakdown for each Pareto design point. (c) Energy and area breakdown for each Pareto design point.

dimension used across different design points due to different memory tiling factors.

Based on these kernel design sweeps, we select Pareto optimal points and use them to further identify optimal HE accelerator designs with a simulator. The simulator models the computations and hardware described in Section VII. It takes as input HE parameter settings from HE-PTune and user defined accelerator microarchitectural parameters (HE kernel implementation, number of PEs, and number of lanes). We swept PEs per accelerator are swept from 2-1024 and lanes per PE from 4-8192. Area is estimated based on architectural parameters while power and performance are derived through simulation. To estimate performance and power for an input DNN, each layer is represented as the number of input/output ciphertexts and partials per output ciphertext. The simulator then maps and multiplexes the number of output neuron ciphertext to available PEs and partials to lanes to derive hardware activity factors. Combining multiplexing and activity factors with HLS latency and power results estimates accelerator performance.

The overall performance of a full inference is modeled on a per-layer granularity; this is because after each layer's linear computations, activations are sent to the client for ReLU and Pooling. Each layer of a DNN is expressed as a series of output neuron computations; from this, we compute the total number of partials per output neuron ciphertext.

To capture the benefits of technology scaling, we report power and area estimates for $5 \mathrm{~nm}$ using foundry-reported scaling factors. Specifically, we use $0.2 \times$ power and $0.22 \times$ area to scale from $40 \mathrm{~nm}$ to $16 \mathrm{~nm}$, based on [42], [43], [61], [63]. From $16 \mathrm{~nm}$ to $5 \mathrm{~nm}$, the power and area scaling factors are $0.32 \times$ and $0.17 \times$, using [56] and recent data from [65]. Together, the power and area scaling factors (40nm to $5 \mathrm{~nm}$ ) are $0.056 \times$ and $0.038 \times$, respectively.

\section{B. Evaluation Results}

1) Intra-Kernel Parallelism: We measure the Pareto optimal design speed ups for HE kernels achieved by the energyoptimal point from the power-latency Pareto frontier. An example design space Pareto frontier for NTT is shown in Figure 10. Recall that these frontiers are used as the cost model for the larger architecture, whose sweeps consider the performance-latency tradeoffs of each kernel. We normalize our speedups to the SEAL library implementation on a $3 \mathrm{GHz}$ Intel SkyLake Xeon processor.
We see modest speedups of individual kernels up to $40 \times$ speedup (average $\approx 10 \times$ speedup) with hardware acceleration. The HE_Add and HE_Mult kernels provide substantial parallelism as the underlying computation consists of elementwise modular additions and multiplications which are easily parallelized. In the case of HE_Rotate, (Swap, Decompose, Compose) and NTT, parts of the underlying computations occur sequentially while others can be parallelized such as the element-wise multiplications and butterfly computations. The key result is that intra-kernel parallelism can reduce $H E$ overhead by roughly one order of magnitude.

2) Inter-Kernel Parallelism: Fortunately, DNNs contain abundant parallelism. With the exception of kernel dependencies within a Lane and the reduction of partial products in PEs, partials and output neurons can be executed in parallel by allocating more hardware resources.

For example, consider CNN Layer6 in ResNet50 $\left(f_{w}=3\right.$, $\left.w=64, c_{i}=c_{o}=64\right)$. If each ciphertext contains a single input channel $(n=4096)$, then all partial products can be computed with 36,864 $\mathrm{HE}$ Mult and $\mathrm{HE}$ Rotate parallel kernel invocations. The partial products for these layers cannot be parallelized since HE_Mult must be performed before HE Rotate under Sched-PA. In HE Rotate, domain conversion from evaluation to coefficient using INTT must be done before decomposition, but the NTT to convert back the domain of decomposed polynomials can be parallelized. As a result, we find that the degree of parallelism that can be exploited at the Lane and PE level is on the order of thousands for ResNet50. The key result is that application interkernel parallelism exposes two to three order of magnitude improvement.

3) Lane and PE DSE: When combined, inter-kernel and intra-kernel parallelism can bridge the remaining 3-4 order of magnitude speedup required to approach plaintext inference speeds on top of HE-PTune and Sched-PA. We conduct a design space exploration to evaluate whether these designs are practical with respect to power and area. Figure 11 shows the results from the design space exploration of ResNet50. The power-latency Pareto points identified in the left-most subplots are the ideal architectures when designing an accelerator tuned only for the model. The Pareto frontiers provide insight into the hardware cost-per-ms tradeoff of inference latency. For ResNet50, we find the Pareto optimal design point requires around $30 \mathrm{~W}$ and $545 \mathrm{~mm}^{2}$ for ResNet50, which are within feasible (albeit high) resource usage of datacenter coproces- 
TABLE VI: Performance of running VGG16 and AlexNet on PT-ResNet50 accelerator. Prt is partials per output CT.

\begin{tabular}{cccccc}
\hline Model & Lat(ms) & Increase & PEs-Lanes & Out CT $\mu$ & (K)Prt $\mu$ \\
\hline ResNet50 & 100 & $0 \%$ & $8-512$ & 147 & 50.5 \\
VGG16 & 215 & $59 \%$ & $16-256$ & 422 & 595 \\
AlexNet & 77 & $28 \%$ & $16-128$ & 475 & 337 \\
\hline
\end{tabular}

sors. The low power density is due to aggressive SRAM tiling to meet aggressively high internal bandwidth targets for NTT units. Upon further analysis, we find that the $128 \times 60$ bit SRAM sizes have a bit density that is $\approx 2.5 \times$ worse than larger $1024 \times 60$ SRAMs, which results in low power density. We also note that the $400 \mathrm{MHz}$ clock target is low for a $5 \mathrm{~nm}$ technology, furthering reducing power density.

To understand the limitations to efficiency and performance of each Pareto design point, Figure 11 shows the Pareto optimal design result for ResNet50 (AlexNet, VGG16, and MNIST exhibit similar trends). Figure 11a shows six design points on the Pareto frontier. Figure $11 \mathrm{~b}$ and Figure 11c show the breakdown of run time and area respectively for these six design points. For extreme low-latency designs (Pareto points 0 and 1), results show that most of the design area goes into small SRAMs which are required to support the enormous internal bandwidth required by NTT units (discussed next). As a result, this leads to impractically large area overheads.

Overall, the results in Figure 11b confirm NTT and reduction ( $\mathrm{HE}$ Rotate) dominate $\mathrm{HE}$ accelerator computation cost. Recall NTT is data intensive and have many small internal SRAMs, which at extreme design points result in high power and area usage. This is compounded by the sheer number of NTT units that operate in parallel, making NTT computations the largest overall area component. We note that even in the most parallel design point considered, the accelerator is compute bound (IO utilization is only 12\%) and NTT remains the primary bottleneck. Moreover, we find that the input and output SRAMs in the architecture do not incur as high of a power and area cost. This means that the input duplication into each PE to support output-stationary computation is relatively inexpensive.

4) Accelerator Generality: Designing a fixed-size accelerator for each DNN model is impractical. Instead, the accelerator can be programmed to support different-sized networks by multiplexing compute logic (PEs and Lanes) to handle different DNN tensor shapes. To quantify the loss associated with underutilized units and imperfect dimension matching, we measure performance loss for different ImageNet models (AlexNet and VGG16) running on the HE accelerator optimized for ResNet50 from Figure 11 (i.e., Point 3).

The performance results are summarized in Table VI. We find both AlexNet and VGG16 experience considerable slowdown relative to their ideal architectures for real-time inference as seen in the Increase column of the table. This is due to the choice of PE and Lane allocations and the differences in layer dimensions. As the table shows, AlexNet and VGG16 layers have a higher average number of output CTs per layer than
ResNet50, and the cost of multiplexing PEs outweighs the cost of poor Lane utilization given the granularity of work (a partial versus an entire output CT). The average number of partials per ciphertext is also much higher. However, ResNet50 is very structured given its use of bottleneck layers many of which have partials per output ciphertext that are divisible by or less than 512, yielding high utilization. Conversely, VGG16's partials per output CT tend to fall just above or below factors of 512 , (e.g., 34, 687, 1086) resulting in lower utilization.

\section{RELATED WORK}

A growing interest in privacy and machine learning has resulted in a body of related work on developing cryptographic solutions. Techniques can be categorized into two groups: HE only [11], [28], [31], [53], or multiparty computation (MPC)based [32], [36], [48], [49]. While each has significantly advanced the field all suffer from either accuracy loss due approximation or high communication/computation overheads.

$H E$ only techniques must address evaluating non-linear functions (e.g., ReLU, MaxPool) using only available addition and multiply operations. CryptoNets [28], CryptoDL [31], and LoLa [11] propose replacing ReLU with low-order polynomials that can readily be computed with HE primitives. However, even with square activations [11], this requires very large HE parameters (e.g., $q \approx 1000$ [28], 440 bits [11], while Cheetah uses 60) for an appropriate noise budget. Moreover, approximate activation functions require re-training [11] and can degrade accuracy [28]. Others propose accelerating HE kernels with accelerators. NTT has been ported to FPGAs [46], [50] and GPUs [2], [3], [17] to speedup polynomial multiplication. Raizi et al [47] propose HEAX to accelerate $\mathrm{HE}$ kernels with FPGAs but only reports two orders of magnitude speedup. While related, the results of HEAX are orthogonal to the contributions of this paper; HEAX uses CKKS (Cheetah uses BFV). Mostly, above works focus on ciphertext-ciphertext multiplication (Cheetah uses ciphertext-plaintext), and targets kernel acceleration (Cheetah focus on the application of DNN inference and general chip architecture).

MPC-based schemes provide an alternative to approximation by combining HE with other security solutions, typically garbled circuit (GC) [32], [36], [40], [44], [48], [49]. Among them, Gazelle is considered the state-of-the-art [32]. Gazelle uses HE for linear layers in the cloud and GC [64] for ReLU and MaxPool on the client. This can significantly improve the latency for small models but results in a severe computational bottleneck in deep models (e.g., ResNet50). Cheetah takes Gazelle as a baseline and focuses on reducing the significant computational overheads of HE.

Other work assumes different threat models with noncryptographic solutions. E.g., [10], [59] use TEEs to isolate private data from untrusted software. Others have looked at limiting information leakage by adding noise (similar to DP) [39]; this provides increased average-case privacy with negligible loss in accuracy. 


\section{CONCLUSiOn}

This paper makes progress on the fundamental roadblocks to adoption of $\mathrm{HE}$ machine learning inference by proposing Cheetah. First, HE-PTune automatically identifies the HE parameter with minimal computational overhead. By fine-grained tuning of HE parameters per each layer, HE-PTune's parameters yield up to $11.7 \times$ performance benefit over the stateof-the-art. Next, Cheetah proposes Sched-PA which yields $10.2 \times$ speedup for dot products in FC and CNN layers in HE. Finally, we propose a custom accelerator architecture to exploit the high application inter- and intra-kernel parallelism. We evaluate the tradeoffs between inference latency and hardware costs and show that combining application level parameter tuning with specialized hardware acceleration can bring HE inference down to practical inference speeds.

\section{REFERENCES}

[1] “"'catapult high-level synthesis"," https://www.mentor.com/hlslp/catapult-high-level-synthesis/, accessed: 04-16-2020.

[2] S. Akleylek, O. Dağdelen, and Z. Y. Tok, "On the efficiency of polynomial multiplication for lattice-based cryptography on GPUs using CUDA," in International Conference on Cryptography and Information Security in the Balkans. Springer, 2015, Conference Proceedings, pp. $155-168$.

[3] A. Al Badawi, B. Veeravalli, C. F. Mun, and K. M. M. Aung, "Highperformance FV somewhat homomorphic encryption on GPUs: An implementation using CUDA," IACR Transactions on Cryptographic Hardware and Embedded Systems, pp. 70-95, 2018.

[4] Apple Differential Privacy Team, "Learning with privacy at scale," Apple Machine Learning Journal, vol. 1, no. 8, pp. 1-25, 2017.

[5] M. Bellare, V. T. Hoang, S. Keelveedhi, and P. Rogaway, "Efficient garbling from a fixed-key blockcipher," in 2013 IEEE Symposium on Security and Privacy. IEEE, 2013, pp. 478-492.

[6] J. W. Bos, K. Lauter, J. Loftus, and M. Naehrig, "Improved security for a ring-based fully homomorphic encryption scheme," in IMA International Conference on Cryptography and Coding. Springer, 2013, pp. 45-64.

[7] J. W. Bos, K. Lauter, and M. Naehrig, "Private predictive analysis on encrypted medical data," Journal of biomedical informatics, vol. 50, pp. 234-243, 2014.

[8] Z. Brakerski, "Fully homomorphic encryption without modulus switching from classical GapSVP," in Advances in cryptology-crypto 2012. Springer, 2012, pp. 868-886.

[9] Z. Brakerski, C. Gentry, and V. Vaikuntanathan, "(Leveled) fully homomorphic encryption without bootstrapping," ACM Transactions on Computation Theory (TOCT), vol. 6, no. 3, p. 13, 2014.

[10] F. Brasser, T. Frassetto, K. Riedhammer, A.-R. Sadeghi, T. Schneider, and C. Weinert, "VoiceGuard: Secure and private speech processing." in Interspeech, 2018, pp. 1303-1307.

[11] A. Brutzkus, R. Gilad-Bachrach, and O. Elisha, "Low latency privacy preserving inference," in International Conference on Machine Learning, 2019, pp. 812-821.

[12] K. Chaudhuri and D. Hsu, "Sample complexity bounds for differentially private learning," in Proceedings of the 24th Annual Conference on Learning Theory, 2011, pp. 155-186.

[13] W.-S. Choi, M. Tomei, J. R. S. Vicarte, P. K. Hanumolu, and R. Kumar, "Guaranteeing local differential privacy on ultra-low-power systems," in 2018 ACM/IEEE 45th Annual International Symposium on Computer Architecture (ISCA), June 2018, pp. 561-574.

[14] F. Chollet et al., "Keras," https://keras.io, 2015.

[15] V. Costan and S. Devadas, "Intel SGX explained." IACR Cryptology ePrint Archive, vol. 2016, no. 086, pp. 1-118, 2016.

[16] W. Dai, "cuhe: Cuda homomorphic encryption library." [Online]. Available: https://github.com/vernamlab/cuHE

[17] W. Dai and B. Sunar, "cuHE: A homomorphic encryption accelerator library," in International Conference on Cryptography and Information Security in the Balkans. Springer, 2015, Conference Proceedings, pp. 169-186.
[18] B. Ding, J. Kulkarni, and S. Yekhanin, "Collecting telemetry data privately," in Advances in Neural Information Processing Systems, 2017. pp. 3571-3580.

[19] L. Ducas and D. Micciancio, "FHEW: bootstrapping homomorphic encryption in less than a second," in Annual International Conference on the Theory and Applications of Cryptographic Techniques. Springer, 2015, pp. 617-640.

[20] C. Dwork, A. Roth et al., "The algorithmic foundations of differential privacy," Foundations and Trends® in Theoretical Computer Science, vol. 9, no. 3-4, pp. 211-407, 2014.

[21] Ú. Erlingsson, V. Pihur, and A. Korolova, "Rappor: Randomized aggregatable privacy-preserving ordinal response," in Proceedings of the 2014 ACM SIGSAC conference on computer and communications security. ACM, 2014, pp. 1054-1067.

[22] J. Fan and F. Vercauteren, "Somewhat practical fully homomorphic encryption.” IACR Cryptology ePrint Archive, vol. 2012, p. 144, 2012.

[23] P. Fenner and E. O. Pyzer-Knapp, "Privacy-preserving Gaussian process regression - a modular approach to the application of homomorphic encryption," 2020.

[24] C. Gentry, "Fully homomorphic encryption using ideal lattices," in Proceedings of the Forty-first Annual ACM Symposium on Theory of Computing, ser. STOC '09. New York, NY, USA: ACM, 2009, pp. 169178. [Online]. Available: http://doi.acm.org/10.1145/1536414.1536440

[25] C. Gentry, "Computing arbitrary functions of encrypted data," Commun. $A C M$, vol. 53, no. 3, pp. 97-105, Mar. 2010. [Online]. Available: http://doi.acm.org/10.1145/1666420.1666444

[26] C. Gentry, S. Halevi, and N. P. Smart, "Fully homomorphic encryption with polylog overhead," in Annual International Conference on the Theory and Applications of Cryptographic Techniques. Springer, 2012, pp. $465-482$.

[27] C. Gentry, A. Sahai, and B. Waters, "Homomorphic encryption from learning with errors: Conceptually-simpler, asymptotically-faster, attribute-based," in Advances in Cryptology-CRYPTO 2013. Springer, 2013, pp. 75-92.

[28] R. Gilad-Bachrach, N. Dowlin, K. Laine, K. Lauter, M. Naehrig, and J. Wernsing, "CryptoNets: Applying neural networks to encrypted data with high throughput and accuracy," in International Conference on Machine Learning, 2016, pp. 201-210.

[29] S. Halevi and V. Shoup, "Algorithms in HElib," in Annual Cryptology Conference. Springer, 2014, pp. 554-571.

[30] K. He, X. Zhang, S. Ren, and J. Sun, "Deep residual learning for image recognition," in Proceedings of the IEEE conference on computer vision and pattern recognition, 2016, pp. 770-778.

[31] E. Hesamifard, H. Takabi, and M. Ghasemi, "CryptoDL: Deep neural networks over encrypted data," arXiv preprint arXiv:1711.05189, 2017.

[32] C. Juvekar, V. Vaikuntanathan, and A. Chandrakasan, "Gazelle: A low latency framework for secure neural network inference," arXiv preprint arXiv:1801.05507, 2018.

[33] J. Katz, A. J. Menezes, P. C. Van Oorschot, and S. A. Vanstone, Handbook of applied cryptography. CRC press, 1996.

[34] A. Krizhevsky, I. Sutskever, and G. E. Hinton, "Imagenet classification with deep convolutional neural networks," in Advances in neural information processing systems, 2012, pp. 1097-1105.

[35] Y. LeCun, L. Bottou, Y. Bengio, P. Haffner et al., "Gradient-based learning applied to document recognition," Proceedings of the IEEE, vol. 86, no. 11, pp. 2278-2324, 1998.

[36] J. Liu, M. Juuti, Y. Lu, and N. Asokan, "Oblivious neural network predictions via MiniONN transformations," in Proceedings of the 2017 ACM SIGSAC Conference on Computer and Communications Security. ACM, 2017, pp. 619-631.

[37] V. Lyubashevsky, C. Peikert, and O. Regev, "On ideal lattices and learning with errors over rings," in Annual International Conference on the Theory and Applications of Cryptographic Techniques. Springer, 2010, pp. 1-23.

[38] D. Malkhi, N. Nisan, B. Pinkas, Y. Sella et al., "Fairplay-secure twoparty computation system." in USENIX Security Symposium, vol. 4. San Diego, CA, USA, 2004, p. 9.

[39] F. Mireshghallah, M. Taram, P. Ramrakhyani, D. Tullsen, and H. Esmaeilzadeh, "Shredder: Learning noise distributions to protect inference privacy," ASPLOS, 2019.

[40] P. Mohassel and Y. Zhang, "SecureML: A system for scalable privacypreserving machine learning," in 2017 IEEE Symposium on Security and Privacy (SP), May 2017, pp. 19-38. 
[41] P. Mohassel and P. Rindal, "ABY3: a mixed protocol framework for machine learning," in Proceedings of the 2018 ACM SIGSAC Conference on Computer and Communications Security. ACM, 2018, pp. 35-52.

[42] NanotechnologyProductsDatabase, "TSMC 20nm Technology." [Online]. Available: https://product.statnano.com/product/6776/tsmc20nm-technology

[43] NanotechnologyProductsDatabase, "TSMC 28nm Technology." [Online]. Available: https://product.statnano.com/product/6777/tsmc28nm-technology

[44] C. Orlandi, A. Piva, and M. Barni, "Oblivious neural network computing via homomorphic encryption," EURASIP Journal on Information Security, vol. 2007, no. 1, p. 037343, 2007.

[45] N. Papernot, M. Abadi, U. Erlingsson, I. Goodfellow, and K. Talwar, "Semi-supervised knowledge transfer for deep learning from private training data," arXiv preprint arXiv:1610.05755, 2016.

[46] T. Pöppelmann, M. Naehrig, A. Putnam, and A. Macias, "Accelerating homomorphic evaluation on reconfigurable hardware," in International Workshop on Cryptographic Hardware and Embedded Systems. Springer, 2015, pp. 143-163.

[47] M. S. Riazi, K. Laine, B. Pelton, and W. Dai, "Heax: An architecture for computing on encrypted data," in Proceedings of the Twenty-Fifth International Conference on Architectural Support for Programming Languages and Operating Systems, ser. ASPLOS '20. New York, NY, USA: Association for Computing Machinery, 2020, p. 1295-1309. [Online]. Available: https://doi.org/10.1145/3373376.3378523

[48] M. S. Riazi, C. Weinert, O. Tkachenko, E. M. Songhori, T. Schneider, and F. Koushanfar, "Chameleon: A hybrid secure computation framework for machine learning applications," in Proceedings of the 2018 on Asia Conference on Computer and Communications Security. ACM, 2018, pp. 707-721.

[49] B. D. Rouhani, M. S. Riazi, and F. Koushanfar, "DeepSecure: Scalable provably-secure deep learning," arXiv preprint arXiv:1705.08963, 2017

[50] S. S. Roy, F. Turan, K. Jarvinen, F. Vercauteren, and I. Verbauwhede, "FPGA-based high-performance parallel architecture for homomorphic computing on encrypted data," in 2019 IEEE International Symposium on High Performance Computer Architecture (HPCA). IEEE, 2019, pp. 387-398.

[51] O. Russakovsky, J. Deng, H. Su, J. Krause, S. Satheesh, S. Ma, Z. Huang, A. Karpathy, A. Khosla, M. Bernstein et al., "Imagenet large scale visual recognition challenge," International journal of computer vision, vol. 115, no. 3, pp. 211-252, 2015.

[52] A.-R. Sadeghi, T. Schneider, and I. Wehrenberg, "Efficient privacypreserving face recognition," in International Conference on Information Security and Cryptology. Springer, 2009, pp. 229-244.

[53] A. Sanyal, M. J. Kusner, A. Gascón, and V. Kanade, "TAPAS: Tricks to accelerate (encrypted) prediction as a service," arXiv preprint arXiv:1806.03461, 2018.

[54] M. Satyanarayanan, "The emergence of edge computing," Computer, vol. 50, no. 1, pp. 30-39, 2017.

[63] S. Wu, C. Y. Lin, S. H. Yang, J. J. Liaw, and J. Y. Cheng, "Advancing foundry technology with scaling and innovations," in Proceedings of
[55] "Simple Encrypted Arithmetic Library (release 2.3.1)," http://sealcrypto. org, Jun. 2018, microsoft Research, Redmond, WA.

[56] A. Shilov, “TSMC's 5nm EUV Making Progress: PDK, DRM, EDA Tools, 3rd Party IP Ready." [Online]. Available: https://www.anandtech.com/show/14175/tsmcs-5nm-euv-processtechnology-pdk-drm-eda-tools-3rd-party-ip-ready

[57] K. Simonyan and A. Zisserman, "Very deep convolutional networks for large-scale image recognition," arXiv preprint arXiv:1409.1556, 2014.

[58] N. P. Smart and F. Vercauteren, "Fully homomorphic SIMD operations," Designs, codes and cryptography, vol. 71, no. 1, pp. 57-81, 2014.

[59] F. Tramer and D. Boneh, "Slalom: Fast, verifiable and private execution of neural networks in trusted hardware," arXiv preprint arXiv:1806.03287, 2018.

[60] F. Tramèr, F. Zhang, A. Juels, M. K. Reiter, and T. Ristenpart, "Stealing machine learning models via prediction APIs." in USENIX Security Symposium, 2016, pp. 601-618.

[61] V. Wang, "TSMC Fights back Intel in Terms of $16 \mathrm{~nm}$ FinFET." [Online]. Available: https://en.ctimes.com.tw/DispNews.asp? $\mathrm{O}=\mathrm{HJY} 1 \mathrm{GE} 204 \mathrm{OGSAA00NK}$

[62] D. Wu and J. Haven, "Using homomorphic encryption for large scale statistical analysis," Technical Report: cs. stanford. edu/people/dwu4/papers/FHESI Report. pdf, Tech. Rep., 2012. Technical Program - 2014 International Symposium on VLSI Technology, Systems and Application (VLSI-TSA), April 2014, pp. 1-3.

[64] A. C.-C. Yao, "How to generate and exchange secrets," in Foundations of Computer Science, 1986., 27th Annual Symposium on. IEEE, 1986, pp. 162-167.

[65] G. Yeap, S. Lin, Y. Chen, H. Shang, P. Wang, H. Lin, Y. Peng, J. Sheu, M. Wang, X. Chen, B. Yang, C. Lin, F. Yang, Y. Leung, D. Lin, C. Chen, K. Yu, D. Chen, C. Chang, H. Chen, P. Hung, C. Hou, Y. Cheng, J. Chang, L. Yuan, C. Lin, C. Chen, Y. Yeo, M. Tsai, H. Lin, C. Chui, K. Huang, W. Chang, H. Lin, K. Chen, R. Chen, S. Sun, Q. Fu, H. Yang, H. Chiang, C. Yeh, T. Lee, C. Wang, S. Shue, C. Wu, R. Lu, W. Lin, J. Wu, F. Lai, Y. Wu, B. Tien, Y. Huang, L. Lu, J. He, Y. Ku, J. Lin, M. Cao, T. Chang, and S. Jang, " $5 \mathrm{~nm}$ cmos production technology platform featuring full-fledged euv, and high mobility channel finfets with densest $0.021 \mathrm{~mm}^{2}$ sram cells for mobile soc and high performance computing applications," in 2019 IEEE International Electron Devices Meeting (IEDM). IEEE, 2019.

[66] S. Zahur, M. Rosulek, and D. Evans, "Two halves make a whole," in Annual International Conference on the Theory and Applications of Cryptographic Techniques. Springer, 2015, pp. 220-250.

[67] J. Zhang, Z. Gu, J. Jang, H. Wu, M. P. Stoecklin, H. Huang, and I. Molloy, "Protecting intellectual property of deep neural networks with watermarking," in Proceedings of the 2018 on Asia Conference on Computer and Communications Security. ACM, 2018, pp. 159-172.

[68] Y. Zhu, G.-Y. Wei, and D. Brooks, "Cloud no longer a silver bullet, edge to the rescue," arXiv preprint arXiv:1802.05943, 2018. 